\title{
Die mikrochirurgische Nervennaht an der Hand
}

\author{
Felix Stang, Erini Liodaki, Peter Mailänder
}

\section{Zusammenfassung}

Die mikrochirurgische Versorgung von Nervenverletzungen an der Hand setzt nicht nur hohe apparative Ausstattung (Operationsmikroskop, Mikroinstrumentarium), sondern auch technische Fertigkeiten und Expertise voraus, da nicht nur die primäre Nervennaht beherrscht werden muss, sondern auch rekonstruktive Eingriffe bei Nervendefekten. Dieser hohe Aufwand ist gerechtfertigt, um schmerzhafte Folgezustände zu vermeiden und regelhaft auch mindestens eine Schmerz-/ Schutzsensibilität wiederherzustellen und bei jungen Patienten auch eine (partielle) motorische Re-Innervation zu erreichen.

\section{Microsurgical Nerve Repair in the Hand}

The microsurgical treatment of nerve injuries of the hand requires a high technical standard (operation microscope, microsurgical instruments) and a high level of expertise, as the neurosurgeon must perform a primary nerve suture and be capable of reconstructing neural defects. This effort is justified, as it can help to avoid painful neuromas and can lead to the restoration of at least some protective sensitivity to pain, as well as partial motor reinnervation.

\section{Einleitung}

Patienten mit Verletzungen an der oberen Extremität und v.a. an der Hand gehören zur täglichen Routine in Krankenhäusern. Aufgrund der engen Anatomie der Hand kommt es auch bei vermeintlichen Bagatellverletzungen (z. B. Schnittwunden) schnell zur Beteiligung funktioneller Strukturen wie Sehnen, Nerven und Gefäßen [1] - allein in Europa und den USA schätzt man ca. $700000 \mathrm{~Pa}-$ tienten pro Jahr mit traumatischen Nervenverletzungen [2]. Gefäß- und Nervenverletzungen bedürfen i.d.R. einer Versorgung durch mikrochirurgische Operationstechniken. Vor allem die Nervenverletzungen können an der Hand dramatische Konsequenzen für die Patienten in Beruf und Alltag haben, da das Feingefühl in den Fingerbeeren, aber auch die Funktion der kleinen Handmuskeln gestört sein können, was erhebliche

OP-JOURNAL 2015; 31: 78-81

(c) Georg Thieme Verlag KG Stuttgart · New York DOI http://dx.doi.org/10.1055/s-0035-1557767
Einschränkungen vor allem bei feinmotorischen Tätigkeiten nach sich zieht.

Die mikrochirurgische Versorgung von Gefäß- und Nervenverletzungen stellt besondere Ansprüche an die Expertise des Operateurs und jahrelanges Training ist Voraussetzung.

\section{Anatomie und Pathologie}

Die Kenntnis des anatomischen Aufbaus eines Nervs ist wichtig, weil die Techniken der operativen Versorgung darauf aufbauen. Ein peripherer Nerv besteht aus Hüllschichten und den eigentlichen Nervenfasern (Axone). Die dazugehörigen Nervenzellen befinden sich im Rückenmark und in den Spinalganglien. Mehrere Nervenfasern sind eingebettet in das sog. Endoneurium und von Perineurium ummantelt. Dieses Gebilde ist ein sog. Faszikel, mehrere Faszikel sind vom Epineurium ummantelt, welches die äußere Schicht eines Nervs darstellt. Die den Nerv versorgenden Blutgefäße sind im Epineurium und Endoneurium eingebettet. Die einzelnen Axone sind von Schwann-Zellen ummantelt, die wie bei einem Stromkabel die Isolationsschicht (sog. Myelin) bilden (Abb.1 a). Die Nervenfaser kann entweder sensibel (afferent, z.B. für Berührungsempfinden der Haut) oder motorisch (efferent) sein (z.B. für Muskeln).

An der Hand findet sich eine besondere Dichte an Nervenfasern, da das taktile Auflösungsvermögen in den Fingerbeeren sehr groß ist (Stichwort Blindenschrift). Insgesamt 3 große Stammnerven, N. radialis (radialer Handrücken), N. medianus (Finger 1-4) und N. ulnaris (Finger 4-5) beteiligen sich an der Innervation der Hand. Jeder Finger besitzt 2 wichtige Nerven und Arterien auf der Beugeseite - sie laufen ulnar und radial der Beugesehnen und sind hier gegenüber z.B. Schnittverletzungen besonders exponiert (Abb.1 b,c). Es werden verschiedene Grade der Nervenverletzung unterschieden. Die häufigste Unterteilung nach Seddon (1943) beschreibt 3 Muster, die in Tab. 1 dargestellt sind.

Die vollständige Durchtrennung eines Nervs führt zu einer aufgehobenen elektrischen Reizleitung des Nervs und eine einfache Naht führt nicht dazu, dass die elektrische Leitfähigkeit wie bei einem Stromkabel wiederhergestellt ist.

Vielmehr kommt es zu einer Degeneration der distal der Verletzung gelegenen Nervenfasern, wobei nur die nervalen Hüllstrukturen und die Schwann-Zellen übrig bleiben (sog. Waller-Degeneration, 1850) und die Nervenzelle muss erst die Nervenfasern durch diesen Anteil nachwachsen lassen (sog. „Nervenregeneration“). Als Richtwert wird eine Wachstumsgeschwindigkeit von ca. $1 \mathrm{~mm} /$ Tag angenommen, dieser Wert liegt in praxi jedoch deutlich niedriger, sodass eine erfolgreiche Regeneration in Abhängigkeit der Läsionshöhe mehrere Monate in Anspruch nehmen kann. Damit die Nervenfasern den richtigen Weg zum Erfolgsorgan (Tastkörperchen, Muskeln) finden, 


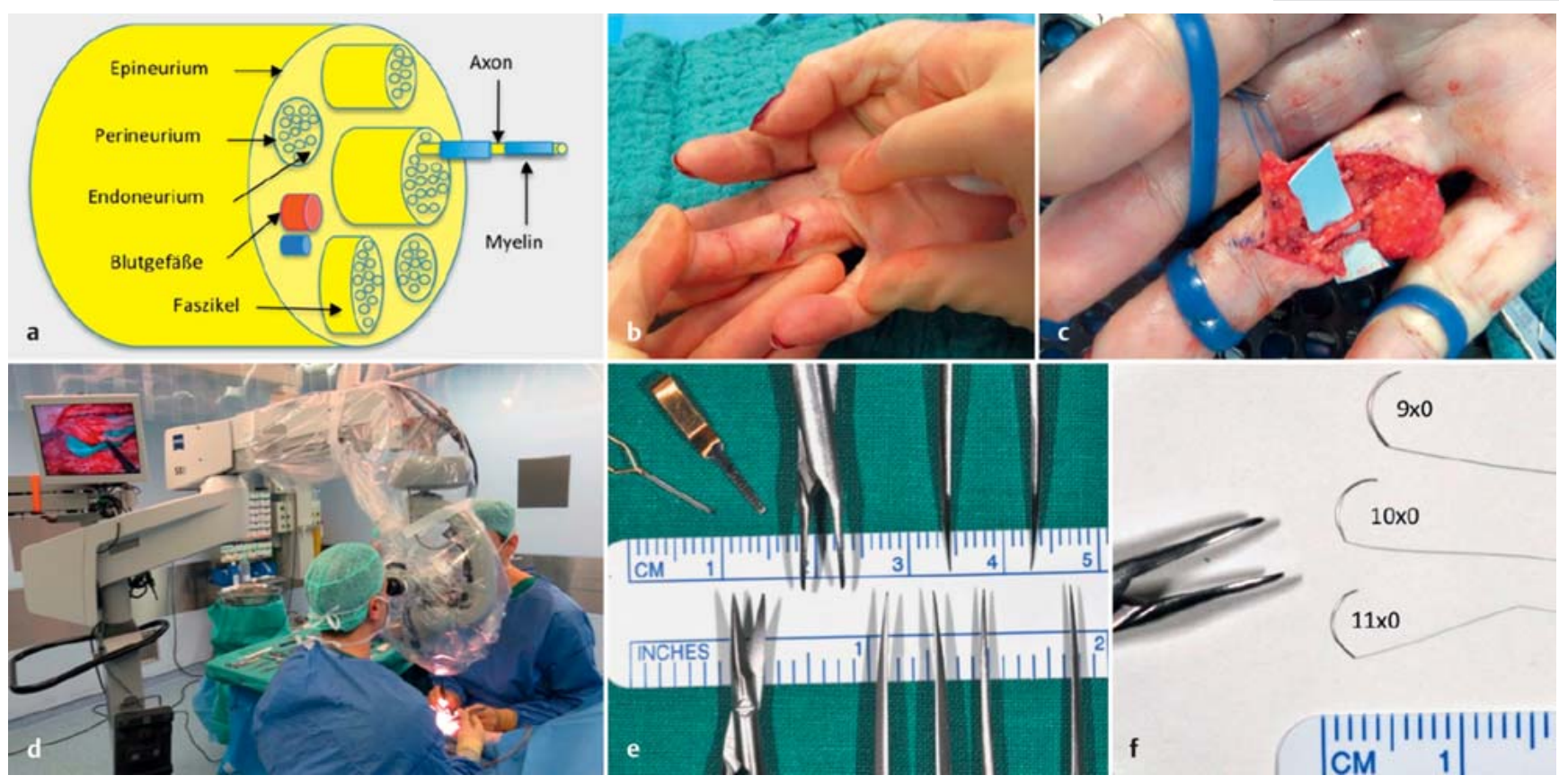

Abb. 1 a bis $\mathbf{f}$ a Schematischer Aufbau eines Nervs mit den verschiedenen Hüllschichten, die Faszikel und Nervenfasern umschließen. b Typische Schnittwunde am Finger mit isolierter Durchtrennung des ulnaren Gefäß-Nerven-Bündels, welches mikrochirurgisch genäht wurde (c). d Aufbau/ Anordnung eines Operationsmikroskops. e Mikrochirurgisches Instrumentarium. f Fadenstärken $9 \times 0$ bis $11 \times 0$ im Vergleich, daneben die Spitze eines Nadelhalters.

muss eine möglichst präzise Naht des durchtrennten Nervs erfolgen, wobei insbesondere darauf geachtet werden muss, die richtige Ausrichtung der Nervenstümpfe (Faszikel) zueinander zu finden. Verletzte Nerven neigen zu Neuromen, einem Knäuel aus ungezielt nachwachsenden Nervenfasern, die sehr schmerzhaft sein können, v.a. bei Berührung und Druck. Daher sollte grundsätzlich immer eine Versorgung von Nervenverletzungen vorgenommen werden, um mindestens das Risiko der Neuromformation zu minimieren, auch wenn bspw. angesichts des Patientenalters eine Regeneration nicht Erfolg versprechend ist.

\section{Technische Voraussetzungen}

Für die mikrochirurgische Versorgung gelten besondere Voraussetzungen. Man braucht ein binokulares Operationsmikroskop (Abb. 1 d), durch das idealerweise Operateur und Assistent, die sich gegenüber sitzen, sehen können. Das monofile, nicht resorbierbare Nahtmaterial ist sehr fein, zwischen $9 \times 0$ und $11 \times 0$ (ca. $0,04-0,01 \mathrm{~mm}$ ), mit entsprechend kleinen Nadeln (Abb. 1 f). Für die Nervenchirurgie werden sog. SpatulaNadeln verwendet, die im Gegensatz zu den runden Gefäßnadeln einen flachen Spitzenschliff haben. Für diese kleinen Nadeln sind natürlich entsprechende Instrumente Voraussetzung (Abb. 1 e). Ein

Tab. 1 Verschiedene Grade der Nervenverletzung, Unterteilung nach Seddon (1943).

\begin{tabular}{ll} 
Neurapraxie & $\begin{array}{l}\text { Nerv ist in Kontinuität bei intakten Hüllstrukturen, jedoch lokale Demyeli- } \\
\text { nisierung bei intakten Nervenfasern; Beispiel: Druckschaden beim Karpal- } \\
\text { tunnelsyndrom oder Quetschverletzungen }\end{array}$ \\
\hline Axonotmesis & $\begin{array}{l}\text { Nerv ist in Kontinuität bei intakten Hüllstrukturen, jedoch Unterbrechung } \\
\text { der Nervenfasern }\end{array}$ \\
\hline Neurotmesis & Vollständige Durchtrennung mit Unterbrechung auch der Hüllstrukturen
\end{tabular}

typisches mikrochirurgisches Instrumentenset besteht aus mehreren Mikropinzetten (Spitze 0,2-0,3 mm), einem Mikronadelhalter (Spitze 0,2-0,4 mm), Mikroscheren, wovon eine i.d. R. gezahnt ist (sog. Millesi-Schere). Die Zahnung sorgt dafür, dass beim Schneiden des Nervs dieser nicht aus der Schere herausrutscht und damit ein sauberer, glatter Schnitt möglich ist. Darüber hinaus beinhaltet ein Sieb typischerweise noch Mikrokanülen und Mikrogefäßklemmen für mikrochirurgische Gefäßnähte. Da die feinen Spitzen der Instrumente sehr empfindlich sind, ist ein entsprechend schonender Umgang mit diesen Instrumenten obligat - idealerweise werden diese auf dem Instrumentiertisch auf Bauchtüchern gelagert, um ein versehentliches Verbiegen der Spitzen auf dem Tisch zu vermeiden. Die Instrumente sollten stets gesäubert angereicht werden, da eingetrocknete Blutreste das Gleiten des Fadens behindern. Dem Ope- rateur müssen die Instrumente präzise in die Hand gelegt werden, da dieser i.d.R. kontinuierlich durch das Operationsmikroskop sieht.

\section{OP-Technik}

Grundvoraussetzungen für Mikrochirurgie jeglicher Art ist ein infektfreier Situs mit der Möglichkeit einer adäquaten Weichteildeckung - auch unter Anwendung plastisch-chirurgischer Rekonstruktionsverfahren.

Die mikrochirurgische Nervennaht („Koaptation“) muss grundsätzlich spannungsfrei erfolgen.

Als Richtwert kann nach Millesi gelten, dass ein $10 \times 0$-Faden beim Knoten in Neutralstellung des darüberliegenden Gelenks nicht reißt. Die Nervenendigungen müssen in gesundes Gewebe zurückgekürzt werden, d.h. alle geschädig- 

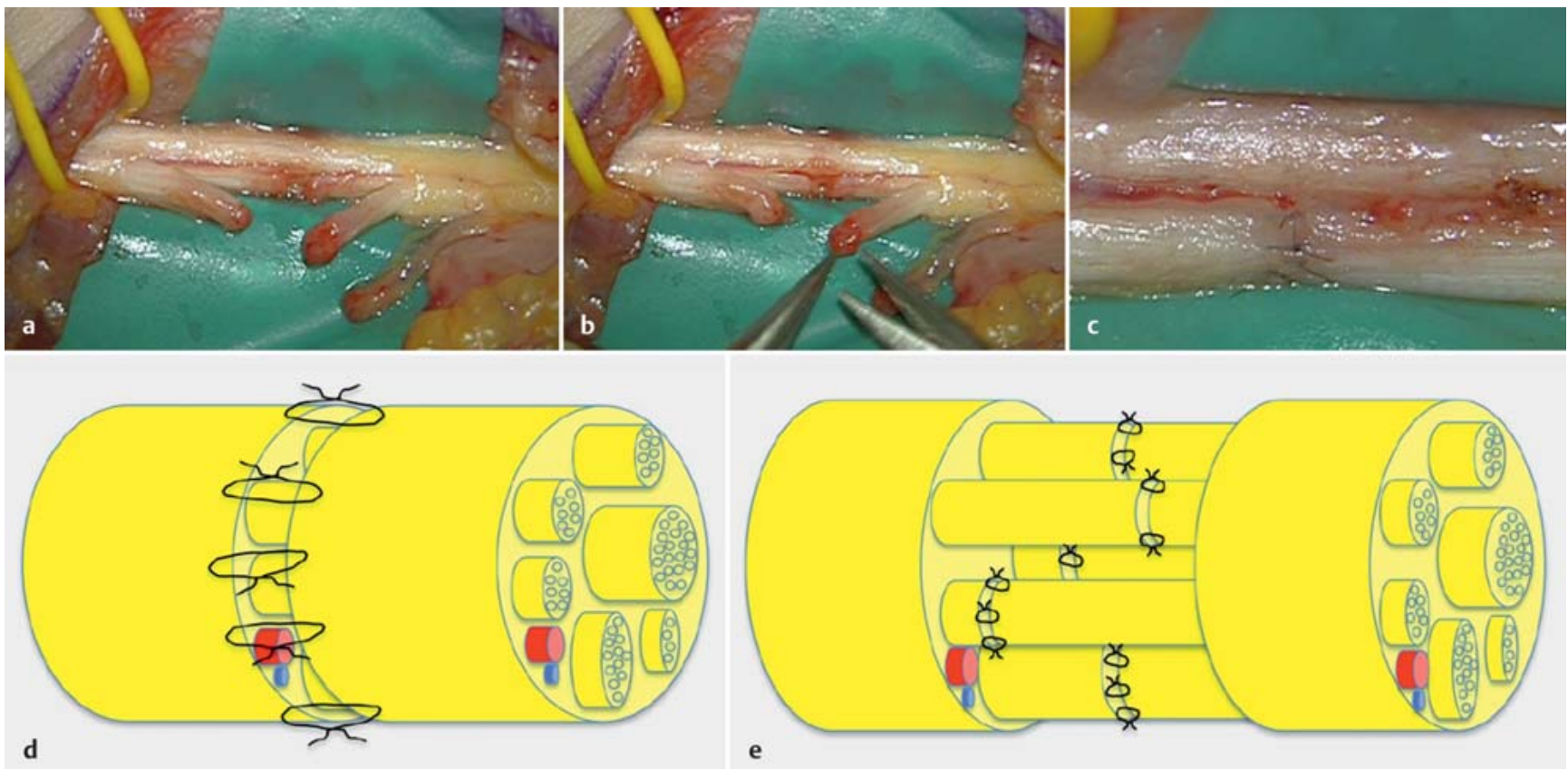

Abb. 2 a bis e a Durchtrennung eines Faszikels des N. medianus am Handgelenk. Die Nervenenden werden débridiert (b) und anschließend mikrochirurgisch mit Einzelknopfnähten koaptiert (c). d Schema der mikrochirurgischen, epineuralen Einzelknopfnaht versus der interfaszikulären, perineuralen Nervennaht (e). Idealerweise sind die Nahtstellen zueinander versetzt, um Vernarbungen auf einer Ebene zu vermeiden - dies findet v. a. bei der Nerventransplantation größerer Nerven Anwendung.

ten oder gequetschten Anteile werden débridiert (Abb. 2 a-c). Es existieren verschiedene Nahttechniken, die 2 häufigsten sind:

\section{Die epineurale Einzelknopfnaht}

(Abb. 2d):

Bei dieser Technik wird ausschließlich

das Epineurium beider Nervenenden miteinander in Einzelknopftechnik vernäht. Zur Anwendung kommt diese Technik bei Nerven mit kleinem Querschnitt bzw. monofaszikulären Nerven, z. B. im Fingerbereich.

2. Die interfaszikuläre Naht (Abb. 2e):

Diese Technik kommt vor allem bei polyfaszikulären Stammnerven mit großem Querschnitt in Betracht. Hierbei werden korrespondierende Faszikel mittels perineuraler Einzelknopfnaht miteinander verbunden. Diese Technik ist deutlich anspruchsvoller, da manchmal eine interfaszikuläre Neurolyse („Aufspleißen“) des Nervs erfolgen muss. Das Risiko der Vernarbung ist deutlich erhöht, was die Nervenregeneration kompromittieren kann.

Ist eine spannungsfreie Nervennaht nicht möglich, müssen Techniken der Nervenrekonstruktion durch Transplantation erwogen werden.

Hierbei können autologe Nerventransplantate, Venen, Venen mit Muskelfül- lung oder artifizielle Nervenröhrchen (Neuragen ${ }^{\circledR}$, Reaxon ${ }^{\circledR}$ ) herangezogen werden. Auch allogene, azelluläre Nerventransplantate (Avance ${ }^{\circledR}$ Nerve Graft) stehen zur Verfügung, werden derzeit jedoch nur an wenigen Zentren verwendet.

\section{Ergebnisse}

Die Ergebnisse von primären Nervennähten sind sehr heterogen und das Outcome unterliegt vielen Variablen [3]. Die wichtigsten Faktoren sind:

1. Patientenalter: Je jünger der Patient ist, desto besser ist die Nervenregenerationspotenz.

2. Höhe der Läsion: Je näher die Verletzung am Erfolgsorgan ist, desto höher sind die Chancen für eine erfolgreiche Re-Innervation.

3. Zeitpunkt der operativen Versorgung: je früher, desto besser.

4. Art der Verletzung bzw. Begleitverletzungen.

5. Betroffener Nerv: bestimmte Nerven regenerieren besser als andere.

6. Qualität der Versorgung bzw. Expertise des Operateurs.

Grundsätzlich muss man davon ausgehen, dass nach einer Nervendurchtrennung keine vollständige Funktionswiederherstellung erfolgt $[4,5]$.
Tab. 2 Sensibilitätskategorisierung nach dem Medical Research Council (MRC 1975).

\begin{tabular}{ll} 
Grad & Qualität der Sensibilität \\
\hline S0 & keine Sensibilität \\
\hline S1 & Schmerzwahrnehmung \\
\hline S2 & $\begin{array}{l}\text { beginnende Schmerzlokalisation } \\
\text { und Berührungsempfinden }\end{array}$ \\
\hline S2+ & Rückgang der Hyperpathie \\
\hline S3 & $\begin{array}{l}\text { Rückkehr der Schmerzlokalisation } \\
\text { und Berührungsempfinden }\end{array}$ \\
\hline S4 & $\begin{array}{l}\text { wie S3 mit Stimuluslokalisation } \\
\text { und 2-PD }\end{array}$ \\
\hline S5 & normale Sensibilität
\end{tabular}

Ausnahmen sind bei Kindern zu verzeichnen, da hier eine bessere Regenerationspotenz auch aufgrund einer besseren kortikalen Plastizität vorhanden ist [6]. Als Kriterium für eine erfolgreiche sensible Regeneration wird u.a. die statische und dynamische 2-Punkt-Diskrimination (2-PD) herangezogen, d.h. der kleinste Abstand im Bereich der Fingerbeere, bei dem 2 (sich bewegende) Berührungspunkte noch getrennt wahrgenommen werden. Der Normwert liegt bei ca. 3-5 mm, unterliegt jedoch enormen interindividuellen Schwankungen, deshalb muss der jeweilige „Referenz- 
wert“ an gesunden Patientenfingern eruiert werden. Dieser Wert fließt in eine Kategorisierung nach dem Medical Research Council (MRC 1975) ein (Tab. 2).

Bei Erwachsenen ist häufig nur eine Wiederherstellung der Schmerzempfindung ohne vollständige taktile 2-PunktDiskrimination zu beobachten; so war bei erwachsenen Fingernerven eine dynamische 2-PD (2-12 mm) nach mikrochirurgischer Naht nur in 68\% aller Fälle nachzuweisen [7]. Eine zufriedenstellende motorische Regeneration bei Verletzungen des $\mathrm{N}$. ulnaris oder $\mathrm{N}$. medianus ist nur in der Hälfte aller Fälle zu verzeichnen [4].

\section{Zusammenfassung}

Die mikrochirurgische Versorgung von Nervenverletzungen setzt hohe apparative und technische Fertigkeiten voraus, da nicht nur die primäre Nervennaht beherrscht werden muss, sondern auch rekonstruktive Eingriffe im Falle eines
Nervendefekts. Dieser hohe Aufwand ist gerechtfertigt, da schmerzhafte Folgezustände vermieden und oft auch mindestens eine Schmerz-/Schutzsensibilität wiederhergestellt werden können. Bei jungen Patienten kann - abhängig von Art und Höhe der Läsion sowie vom Patientenalter - weiterhin auch eine (partielle) Rückkehr der ausgefallenen Muskelfunktionen erwartet werden.

\section{Literatur}

${ }^{1}$ Scholz T, Krichevsky A, Sumarto A et al. Peripheral nerve injuries: an international survey of current treatments and future perspectives. J Reconstr Microsurg 2009; 25: 339-344

2 Yao L, Billiar KL, Windebank AJ et al. Multichanneled collagen conduits for peripheral nerve regeneration: design, fabrication, and characterization. Tissue Eng Part C Methods 2010; 16: 1585-1596

${ }^{3}$ Mafi P, Hindocha S, Shital M et al. Advances of peripheral nerve repair techniques to improve hand function: a systematic review of literature. Open Orthop J 2012; 6: 60-68

${ }^{4}$ Ruijs AC, Jaquet JB, Kalmijn S et al. Median and ulnar nerve injuries: a meta-analysis of predictors of motor and sensory recovery after modern microsurgical nerve repair. Plast Reconstr Surg 2005; 116: 484-494
${ }^{5}$ Lundborg G, Rosén B. Hand function after nerve repair. Acta Physiol (Oxf) 2007; 189: 207-217

${ }^{6}$ Birch R, Achan P. Peripheral nerve repairs and their results in children. Hand Clin 2000; 16 : 579-595

${ }^{7}$ Mailänder P, Schaller E, Berger A et al. Rückkehr der statischen und beweglichen Zweipunktediskriminierung nach Nervenwiederherstellung. Handchir Mikrochir Plast Chir 1989; 21: 299-301

\section{Dr. med. Erini Liodaki}

Assistenzärztin

Prof. Dr. med. Peter Mailänder

Chefarzt

Dr. med. Felix Stang

Leitender Oberarzt

Klinik für Plastische, Rekonstruktive Chirurgie, Handchirurgie Intensiveinheit für Schwerbrandverletzte

Universitätsklinikum Schleswig-

Holstein, Campus Lübeck

Ratzeburger Allee 160

23538 Lübeck

felix.stang@uksh.de 